\title{
Visualization Tool for Atomic Models (VITAL): A Simple Visualization Tool for Materials Predictions
}

Steven Kauwe, Yucheng Yang, Taylor Sparks

Submitted date: 07/09/2019 - Posted date: 09/09/2019

Licence: CC BY-NC-ND 4.0

Citation information: Kauwe, Steven; Yang, Yucheng; Sparks, Taylor (2019): Visualization Tool for Atomic Models (VITAL): A Simple Visualization Tool for Materials Predictions. ChemRxiv. Preprint.

This preprint describes the first version of VITAL, a tool used to visual materials predictions. Instructions for use are outlined and a few basic examples are shown. The code for this tool is included as a zip file and archived version is linked in the text. A GitHub repository is also linked for the most up-to-date version of VITAL.

File list (2)

Simple_visualization_of_materials_predictions.pdf (2.61 MiB) view on ChemRxiv • download file 


\title{
VIsualization Tool for Atomic modeLs (VITAL): A simple visualization tool for materials predictions.
}

\author{
Steven K. Kauwe, Yucheng Yang, and Taylor D. Sparks \\ August 2019 \\ Materials Science \& Engineering, University of Utah, Salt Lake City, UT, 84112. \\ email: sparks@eng.utah.edu
}

\begin{abstract}
In this short communication we seek to demonstrate the "Visualization Tool for Atomic modeLs (VITAL)" that we have created to help with the visualization of machine learning generated materials property predictions. This tool uses interactive figures to allow the user to explore how chemical composition, featurization, and material properties interact with each other. It also allows for composition-based exploration of clustering. Although this tool is in early phases, we hope to expose the community to this idea and are eager to receive important feedback from the community. We also briefly demonstrate some basic uses of this tool and highlight how to download and get started with your own data.
\end{abstract}

\section{Introduction}

The ability to accurately and efficiently predict materials properties would likely have a drastically positive impact on human society. This desire aligns with the core tenet of materials science which is to exploit structure-process-property relationships for the design of better materials for a given application. Incredible resources have been poured into this task. As an example, Materials Genome Initiative-related projects funded by the Federal Government in the first three years alone were in excess of $\$ 250$ million USD [1]. Similarly, the United States Department of Energy budgeted for $\$ 65$ million USD for materials discovery and another $\$ 13$ million for computational materials research for the fiscal year of 2019 [2]. Even with this massive investment of money, time, and human brain power, it would all be justified by the realization of even a few breakthrough materials or manufacturing techniques.

One area of promise is that of materials simulation. Improvements in computing resources and the democratization of powerful computing tools has lead to wide spread calculation of materials properties at various length and time scales. For example, density functional theory (DFT) calculations, molecular dynamics, phase field models and more can simulate properties and experiments at greater speed and lower cost than many experimental approaches. However, these techniques are still inefficient, in that they must approximate physical reality then brute force those approximations into numerically stable solu- tions. Our understanding of both the physics and the computational frameworks themselves are an incredible achievement. However, we are still beholden to many drawbacks regarding these simulations. For example, researchers have struggled to address the fact that there is no clear way to know whether a configuration will be physically realizable. In addition, computation time scales poorly with accuracy and as a result any given simulation can take hours to weeks. Finally, the functional size of simulated systems is often very small in order to stay within reasonable computational constraints.

Despite these well-known limitations, DFT has been and is well positioned as an effective tool for materials discovery [3]. DFT and other high-throughput techniques, are increasingly important aspects of materials science research because they form relatively large databases of calculated properties whereas experimental databases are much smaller and heterogeneous [4]. Consequentially, data-driven materials exploration has experienced incredible growth. Material property prediction using chemical composition and/or structure-based features can be vectorized in a way that is well-suited for classic machine learning techniques. Moreover, the abundance of data that has been generated over the last decade has enabled sufficient training material for end-to-end learning. Thus far, the results of these studies have been positively received, and many researchers have reported the ability of machine learning models to predict various materials properties $[5,6,7,8,9, ?, 10,11,12$, $13,14,15,16,17,12,18]$. As these techniques be- 
come more and more common, the community and its respective researchers are likely to be increasingly exposed to work that use these data-driven tools.

\section{Motivation}

Along with an increased interest in the area of machine learning predicted properties has come a number of growing pains as the materials informatics community adopts best practices. Issues range from inadequate code deployment, to poor reproducibility, overfitting of models, incomplete data wrangling (duplicate entries etc.), insufficient training data, violations in the independent and identically distributed assumption, or simply the inability to transfer learning from training set to reality. Many of the problems related to the data itself stem from the fact that datadriven techniques tend to rely on large datasets, yet traditional materials scientists are ill-equipped with the tools necessary to visualize and identify problems and opportunities in the data itself.

A good example of the need for visualization can be seen in the field of thermoelectrics. A variety of exciting applications become unlocked as thermoelectric efficiency increases. Many researchers have traditionally assumed that the thermoelectric figure of merit, $z T$, will continue to increase as scientists employ new methods of reducing thermal conductivity. However, using a novel visualization of a large thermoelectric materials dataset, Gaultois and Sparks were able to show that achieving a $z T$ of even 4 is highly unlikely based on the Seebeck coefficient of known materials and the ability to ability to reduce lattice thermal conductivity [19]

Visualization of machine learning model output which accounts for chemistry and elemental properties is particularly troublesome. It is unreasonable to expect most researchers, even those employing these tools, to have the type of intimate knowledgeable required to fully understand model predictions. As a result, tools that predict materials properties using data-driven techniques have become common, but the materials science community has not fully embraced this paradigm. We believe this is in part due to the almost mystical nature regarding these machine learned models and their predictions. To those who are not initiated to the field of data-driven materials science, the so called "black box" nature of these model are in a way the antithesis of scientific effort.

However, we feel this need not be the case. While the exact reasoning and behavior and of any given model may not be known, there are often physically reasonable patterns that emerge from these models. To this end, we decided to build the VIsualization Tool for Atomic modeLs (VITAL), with hopes that interactive visualization may help introduce confidence for potential model users. We also hope that VITAL will allow for users to identify problems and/or opportunities in their datasets and models for further improvement which would not be obvious otherwise. With this in mind, we demonstrate how a simple tool can help with the visualization of the interactions between chemical composition, featurization choices, and model predictions.

In particular, we are motivated to create a visualization tool that helps illustrate how these machine learning models work with respect to the data itself. This tool is built in a way that allows researchers to interact with the data by selecting individual elements and seeing how they behave in regards to clustering of similar compositions, the overall predicted values, as well as the error associated with each prediction and all predictions containing a given element.

\section{Usage}

Vital is built with the assumption that a researcher has materials property predictions they would like to visualize and interact with. Using these predictions, this tool will process the predictions, and features if they are available, to generate an interactive interface for the researcher to use. The github repository contains the most recent version of this tool. Instructions for the most recent version can be found in the README.md file. For the archived version we provide instructions below and a sample image of the full tool in Figure 1.

\subsection{Getting setup}

The archived version of this tool [20] is available via zip file on figshare. This should be unzipped into the desired directory. After unzipping, you will find a folder titled "user_data". Your predictions, and your features if desired, can be placed into this folder. This folder also contains example predictions generated from band gap data [21]. These are supplied to illustrate the formatting required for the program to execute.

\subsubsection{Format your files}

In order to visualize your predictions, you will need a comma separated value (CSV) file with the chemical formula in the first column, actual values in the second column and the predicted values in the third column. The three columns of the first row of the CSV file should have cell values: "formula", "actual", and "predicted". Similarly, if you are including a features file, you need a CSV with the chemical formula in the first column, then your tabulated feature in the subsequent columns. 


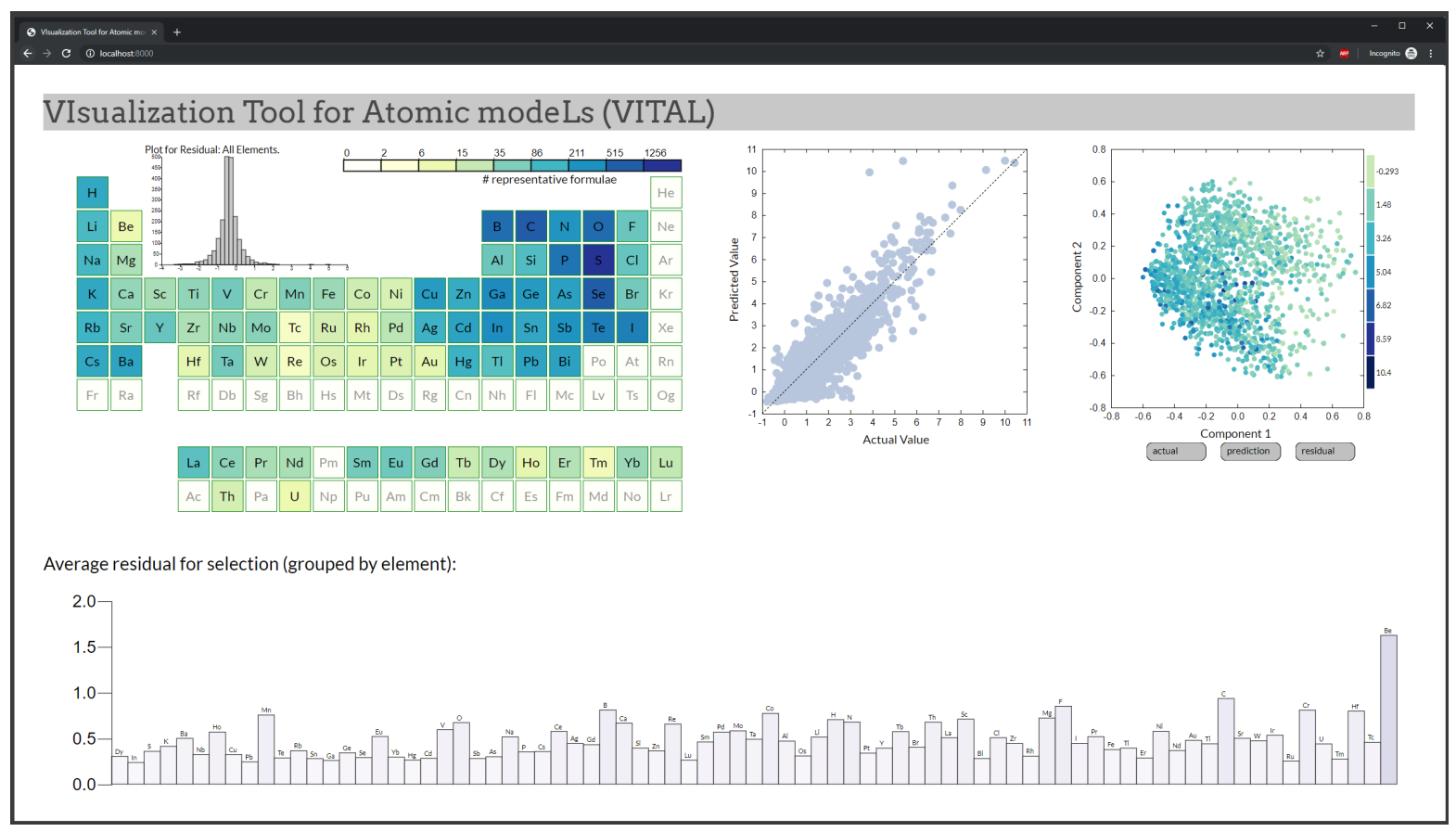

Figure 1: A preview of VITAL. The periodic table interacts with the other plots to help visualize the behavior of elemental presence for the prediction of materials properties using machine learning models.

\subsubsection{Process your data}

In order to process the data, you will simply need to run the python file "main.py" and point it towards the location of your data. This script will do the pre-processing necessary for the visualization of your data. The python script can be run by using your terminal or the command prompt using the command:

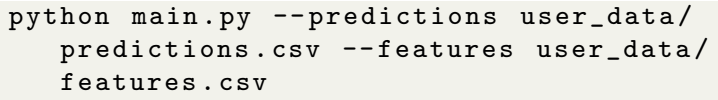

After this script completes you are now ready to load up the tool.

\subsubsection{Start a local server}

The tool will run on a local server and you will interact with it using your browser. Once you have python installed, opening a server is simple. There are two steps you will need to take. The first step is navigating to the correct directory. If you process the data, your terminal/command prompt should be in the main directory, C: $\backslash$ Examplepath $\backslash$ VITAL. From this directory you can type cd js_code and hit enter. You should now be in the directory, C: $\backslash$ Examplepath $\backslash$ VITAL $\backslash j s_{-}$code. From here, you can now use python to open a server with the following command:

python -m http.server 8000
This will open a server on port 8000. We now need to got to this location from our browser and we will be ready to visualize.

\subsubsection{Use tool from browser}

You can now open up the tool from within your favorite browser. This can be done by simple going to the url http://localhost:8000/

\subsection{Basic functionality}

\subsubsection{Visualize elemental prevalence}

The most basic use of this tool is to visualize elemental prevalence. This is achieved by encoding prevalence directly onto our interactive periodic table (Figure 2). This allows us to understand how well balanced our data is. We can also use this periodic table to interact with the other data exploration charts.

\subsubsection{Explore element behavior}

Upon hovering or selecting an element there are two charts that dynamically respond. The first chart shows the actual versus predicted values for a machine learning model trained on band gap. By plotting these values against each other, we show how well our machine learning predictions line up to the real values. Ideal performance lies on a $45^{\circ}$ line. Divergence from this line signifies that some residual error is associated with our predictions. We can quickly hover over 


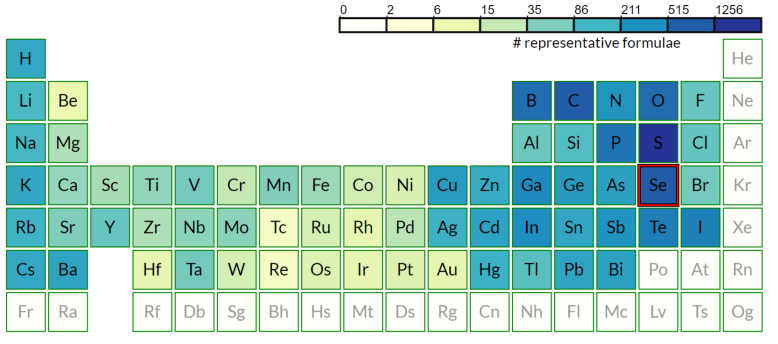

\begin{tabular}{|l|l|l|l|l|l|l|l|l|l|l|l|l|l|l|l|}
\hline La & Ce & Pr & Nd & Pm & Sm & Eu & Gd & Tb & Dy & Ho & Er & Tm & Yb & Lu \\
\hline \hline Ac & Th & Pa & U & Np & Pu & Am & C & Bk & Cf & Es & Fm & Md & No & Lr \\
\hline
\end{tabular}

Figure 2: Periodic table showing a heat map for elemental prevalence based on formulae present in the dataset.

different elements to see whether any are systematically over or under predicted. In Figure 3 we show the behavior of our model with respect to predictions containing Se. The visualization allows for a user to clearly see that, as a rule, compositions with Se tend to have lower band gap.

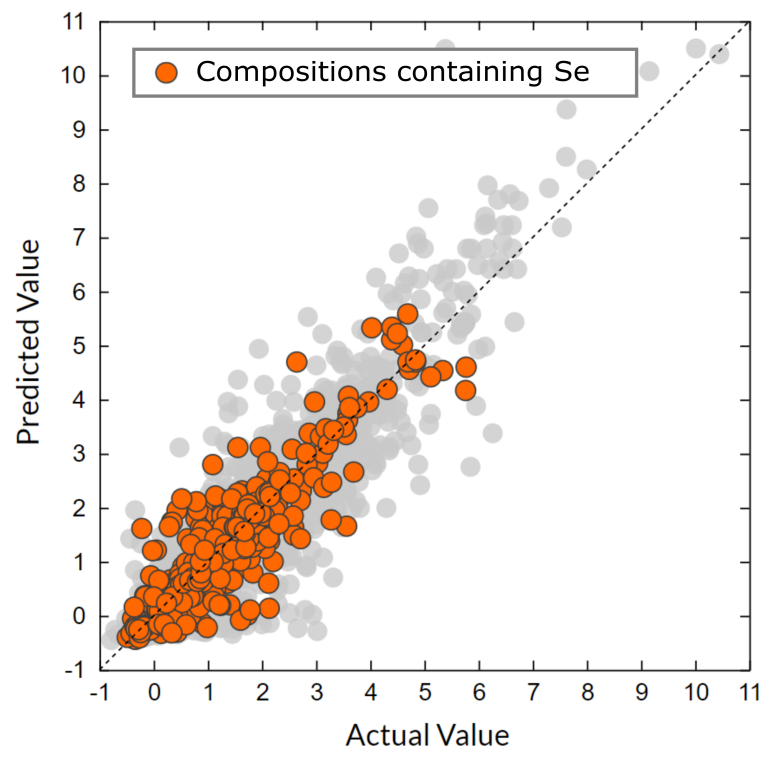

Figure 3: We can explore trained-model behavior by dynamically choosing which elements to look at on the actual versus predicted plot.

\subsubsection{Understand featurization and cluster- ing behavior}

An important aspect of model performance is the features that the model is able to train from. For many algorithms that use gradient descent, such as support vector machines, if is often useful to scale these features. These same scaled features can also be used in principle component analysis (PCA) to try to deter- mine which directions through our feature space best represent the data. In other words, PCA outputs linear combinations of the features, called components, that represent the directions in feature space with the most variance [22].

We use PCA on these scaled features to generate Figure 4, plotting the data on the the two most important components. We also use color to visullly encode information. There are three button you can toggle (not shown) which allow you to switch between the actual property values, the predicted property values, and the residual values. The actual values are currently shown. That said, you will notice in Figure 4 that there is structure to these values and the data itself. Interestingly, we can see that compositions containing the element Se are all grouped towards the upper-right corner. Although it is not shown, this type of clustering is fairly typical across the elements. A better clustering visualization might use t-Distributed Stochastic Neighbor Embedding (tSNE) allowing us to perform non-linear dimensionality reduction [23]. However, in this iteration of VITAL we have chosen to ignore that option due to lengthy computation times and hyper-parameter sensitivity.

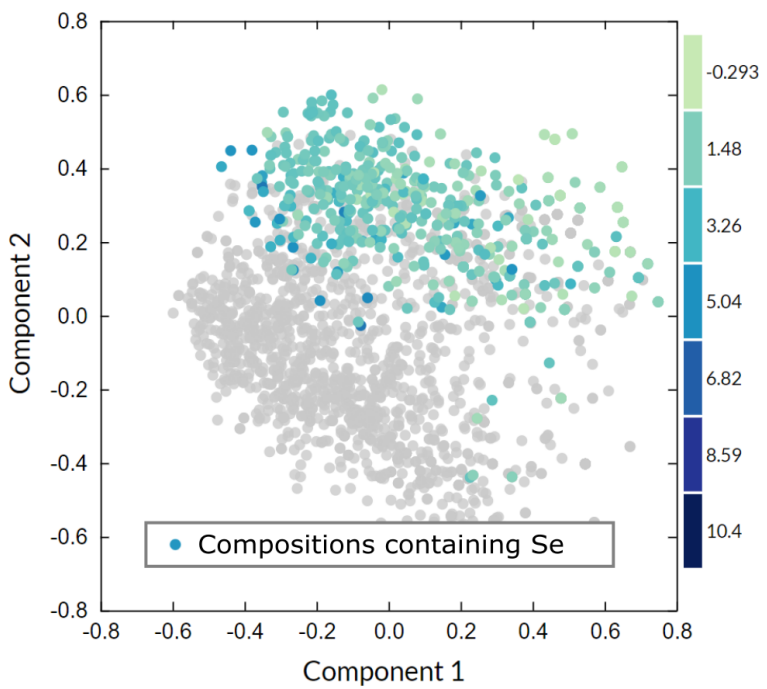

Figure 4: We can use PCA to project our highdimensional feature space down into two-dimensions. We can then visualize how elemental prevalence behaves in this space. For the case of band gap we can see increasing properties as we move towards the bottom-left. We can also see moderate clustering of compositions containing Se.

\subsubsection{Look for patterns in residual}

Finally, a brushing action has been added to the PCA plot that is attached to the residual values of the data. 
Figure 5 shows the PCA plot with residual values encoded with color. Using this as a guide, the user can can explore desired regions of the feature space to see how averaged elemental residual looks.

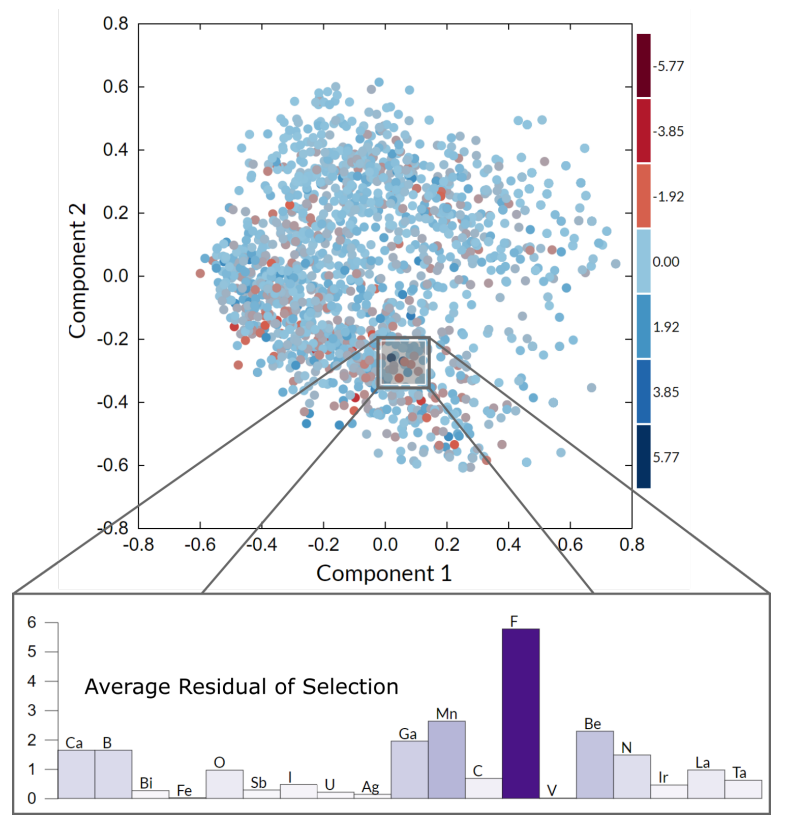

Figure 5: Interactive brush over the PCA plot allows a user to explore average residual over a given selection using color encodings to scale with residual.

\section{Conclusion}

This tool, VITAL, is in early development. We are constantly seeking input for additional features or improvements. As such we have to provided a link to our GitHub repository that provides the most up-to-date version. We have also included an archived version of the code to go with the paper. Ultimately, we hope to host this tool online for anyone to use. Until then, this tool can be downloaded for use on your local computer.

\section{Acknowledgements}

The authors gratefully acknowledge support from NSF CAREER Award DMR 1651668.

\section{References}

[1] Office of Science \& Technology Policy, "Fact sheet: The materials genome initiative - three years of progress." https://www.mgi.gov/ sites/default/files/documents/materials_ genome_initiative_-_three_years.pdf, 2014-06-19. [Online; accessed 4-Sep-2019].

[2] Office of Chief Financial Officer, "Department of energy fy 2020 congressional budget request." https://www.energy.gov/sites/prod/files/ 2019/04/f61/doe-fy2020-budget-volume-4 . pdf, 2019-03. [Online; accessed 4-Sep-2019].

[3] A. Jain, K. A. Persson, and G. Ceder, "Research update: The materials genome initiative: Data sharing and the impact of collaborative ab initio databases," APL Materials, vol. 4, no. 5, p. $053102,2016$.

[4] R. Seshadri and T. D. Sparks, "Perspective: interactive material property databases through aggregation of literature data," APL Materials, vol. 4, no. 5, p. 053206, 2016.

[5] S. K. Kauwe, T. Welker, and T. D. Sparks, "Extracting knowledge from dft," 2018.

[6] A. O. Oliynyk, E. Antono, T. D. Sparks, L. Ghadbeigi, M. W. Gaultois, B. Meredig, and A. Mar, "High-throughput machine-learningdriven synthesis of full-heusler compounds," Chemistry of Materials, vol. 28, no. 20, pp. 73247331, 2016.

[7] G. Hautier, C. C. Fischer, A. Jain, T. Mueller, and G. Ceder, "Finding nature's missing ternary oxide compounds using machine learning and density functional theory," Chemistry of Materials, vol. 22, no. 12 , pp. 3762-3767, 2010.

[8] A. Mansouri Tehrani, A. O. Oliynyk, M. Parry, Z. Rizvi, S. Couper, F. Lin, L. Miyagi, T. D. Sparks, and J. Brgoch, "Machine learning directed search for ultraincompressible, superhard materials," Journal of the American Chemical Society, vol. 140, no. 31, pp. 9844-9853, 2018.

[9] T. D. Sparks, M. W. Gaultois, A. Oliynyk, J. Brgoch, and B. Meredig, "Data mining our way to the next generation of thermoelectrics," Scripta Materialia, vol. 111, pp. 10-15, 2016.

[10] P. Raccuglia, K. C. Elbert, P. D. Adler, C. Falk, M. B. Wenny, A. Mollo, M. Zeller, S. A. Friedler, J. Schrier, and A. J. Norquist, "Machinelearning-assisted materials discovery using failed experiments," Nature, vol. 533, no. 7601, p. 73, 2016.

[11] S. K. Kauwe, J. Graser, A. Vazquez, and T. D. Sparks, "Machine learning prediction of heat capacity for solid inorganics," Integrating Materials and Manufacturing Innovation, vol. 7, no. 2, pp. 43-51, 2018. 
[12] J. Graser, S. K. Kauwe, and T. D. Sparks, "Machine learning and energy minimization approaches for crystal structure predictions: A review and new horizons," Chemistry of Materials, vol. 30, no. 11, pp. 3601-3612, 2018.

[13] V. Tshitoyan, J. Dagdelen, L. Weston, A. Dunn, Z. Rong, O. Kononova, K. A. Persson, G. Ceder, and A. Jain, "Unsupervised word embeddings capture latent knowledge from materials science literature," Nature, vol. 571, no. 7763 , p. 95 , 2019 .

[14] S. K. Kauwe, T. D. Rhone, and T. D. Sparks, "Data-driven studies of li-ion-battery materials," Crystals, vol. 9, no. 1, p. 54, 2019.

[15] S. Kauwe, J. Graser, R. Murdock, and T. Sparks, "Can machine learning find extraordinary materials?," 2019.

[16] O. Isayev, C. Oses, C. Toher, E. Gossett, S. Curtarolo, and A. Tropsha, "Universal fragment descriptors for predicting properties of inorganic crystals," Nature communications, vol. 8, p. $15679,2017$.

[17] L. Ward, A. Agrawal, A. Choudhary, and C. Wolverton, "A general-purpose machine learning framework for predicting properties of inorganic materials," npj Computational Materials, vol. 2, p. 16028, 2016.

[18] T. Xie and J. C. Grossman, "Crystal graph convolutional neural networks for an accurate and interpretable prediction of material properties," Physical review letters, vol. 120, no. 14, p. 145301, 2018.

[19] M. W. Gaultois and T. D. Sparks, "How much improvement in thermoelectric performance can come from reducing thermal conductivity?," $A p$ plied Physics Letters, vol. 104, no. 11, p. 113906, 2014.

[20] S. K. Kauwe, Y. Yang, and T. D. Sparks, "VIsualization Tool for Atomic modeLs (VITAL) v1," 92019.

[21] Y. Zhuo, A. Mansouri Tehrani, and J. Brgoch, "Predicting the band gaps of inorganic solids by machine learning," The journal of physical chemistry letters, vol. 9, no. 7, pp. 1668-1673, 2018.

[22] I. Jolliffe, Principal component analysis. Springer, 2011.

[23] L. v. d. Maaten and G. Hinton, "Visualizing data using t-sne," Journal of machine learning research, vol. 9, no. Nov, pp. 2579-2605, 2008. 
Other files

VITAL.zip (1.43 MiB)

view on ChemRxiv • download file 